\title{
Photometric long-term variations and superhump occurrence in the Classical Nova RR Pictoris
}

\author{
Irma Fuentes Morales* \\ Universidad de Valparaíso, Chile \\ E-mail: irma. fuentes@uv.cl \\ Nikolaus Vogt \\ Universidad de Valparaíso, Chile \\ E-mail: nikolaus.vogteuv.cl

\section{Claus Tappert} \\ Universidad de Valparaíso, Chile \\ E-mail: claus.tappert@uv.cl \\ Linda Schmidtobreick \\ European Southern Observatory, Chile \\ E-mail: 1schmidtaeso.org
}

\begin{abstract}
We present an re-analysis of all available time-resolved photometry from the literature for the classical nova RR Pictoris. The hump light curve phased with the orbital period shows significant variations over the last 42 years in shape and amplitude which possibly are caused by long-term variations in the disc structure. Additionally, we found evidence for the presence of superhumps in 2007, with the same period ( $\sim 9 \%$ longer than the orbital period), as reported earlier by other authors from observations in 2005. Possibly, superhumps arise quickly in RR Pic, but are sporadic events, because in all the other observing runs analysed no significant superhump signal was detected. We also determined an actual version of the Stolz-Schoembs relation between superhump period and orbital period, analysing separately dwarf novae, classical novae and nova-like stars, and conclude that this relation is of general validity for all superhumpers among the cataclysmic variables (CVs), in spite of small but significant differences among the sub-types mentioned above. We emphasize the importance of such a study in context with the still open question of the interrelation between the different sub-classes of CVs, crucial for our understanding of the long-term CV evolution.
\end{abstract}

The Golden Age of Cataclysmic Variables and Related Objects IV

11-16 September, 2017

Palermo, Italy

\footnotetext{
* Speaker.
} 


\section{Introduction}

Cataclysmic variables (CVs) are close interacting binary systems where a late-type dwarf star fills its Roche lobe and transfers mass towards the white dwarf (WD) primary component. In case of a weak -or absent- magnetic field of the WD, the transferred material from the secondary star is accumulated forming an accretion disc around the WD. Once the accumulated mass on the primary reaches a critical value, the surface temperature of the WD increases and triggers nuclear fusion reactions producing a thermonuclear runaway, which is known as a nova eruption. CVs that underwent a nova eruption are called classical novae (for details see [28]).

One of the brightest and nearest novae known is RR Pictoris (distance $d=388 \pm 88 \mathrm{pc}$; [18]). Its nova eruption was discovered by [5] at its maximum brightness $V=1.2$ mag in 1925. Currently the stellar remnant appears to be in a state of quiescence, with $V \sim 12.7$ mag. The photometric light curves of RR Pic show as a permanent feature a periodic hump of $P_{\text {orb }}=3.48 \mathrm{~h}$, which has been identified as the orbital one [24]. Additionally, they have undergone several changes and transient features over the decades since its eruption, showing eclipse-like characteristics [27], 15 min periodicities [6], QPOs and positive superhumps [19]. In a recent work [26] derived a more precise orbital ephemeris from all available photometric humps observed in the past five decades. They furthermore found variations in the $\mathrm{O}-\mathrm{C}$ diagram that could imply the presence of a third body.

The motivation for the present investigation was the discovery already mentioned of an additional period that is 8.6 per cent longer than the orbital one, which was interpreted as a superhump period $\left(P_{\mathrm{sh}}\right)$ by [19]. The presence of superhump in classical novae can be used as a tool to understand the state of the system in general, and of the disc properties decades after the nova eruption.

In this work we present a re-analysis of all available photometric data of RR Pic after its classical nova explosion observations. We also add new data taken during 2013-2014 and study the orbital hump light curves during the past decades in a systematic way. Furthermore we perform a methodical search for superhumps in RR Pic and compare our results with those known for other classical novae, nova-like stars and dwarf novae.

\section{Compilation of historical light curves and new observations}

The light curves of RR Pic analysed are composed of published observations made by different authors, complemented by new data obtained in 2013 and 2014. An observing log for hitherto unpublished data is given as on-line supplementary data associated to [1]. In general, the following campaigns were analysed:

- UBV photometry observed by [24] during four nights in December 1972 and three nights of white light data in January 1974. All these observations have covered more than one orbital cycle.

- High-speed photometry in white light of [27] from 1972 to 1984 over 23 nights. In eight of them at least one orbital period was covered.

- Light curves in five nights observed in January 1980 by [3] in the V band. In all of them at least one complete orbital cycle was observed. 
- [6] obtained UBV photoelectric photometry in five nights in October 1982. We analysed the published B differential band light curves.

- The Center Backyard Astrophysics (CBA) kindly provided us with additional unpublished differential photometry in white light of RR Pic, obtained in a total of 50 nights between 1999 and 2007 by different amateur astronomers.

- Differential photometric data obtained by [19] during the first semester of 2005, covering 18 complete orbital cycles.

- In addition we obtained new V band photometry in 81 nights between 21 November 2013 and 16 March 2014, using the robotic $40 \mathrm{~cm}$ telescope at the Remote Observatory Atacama Desert (ROAD), situated in San Pedro de Atacama, Chile [4]. The integration time was 30 seconds with a cadence of 2-3 minutes.

\section{Results}

\subsection{Variations of orbital light curve}

We used the PERIOD04 software [7], which is based on Discrete Fourier Transform (DFT) to search peridiocities in the light curves. In all campaigns the respective periodogram showed the known orbital frequency as the dominant peak close to $f_{0}=6.895 \mathrm{c} / \mathrm{d}$, corresponding to $P_{\text {orb }}=$ $3.48 h$. The orbital phase of each campaign was calculated using the long-term ephemeris of the orbital hump maximum:

$$
H J D(\max )=2438815.3664(15)+0^{d} .145025959(15) E
$$

derived by [26]. The mean phased-folded light curves were computed by averaging the data points and are shown in Fig. 1.

The orbital light curve properties of RR Pic during the past 42 years can be summarised in the following way: the dominant orbital hump was always present, but with variable amplitude between $0.28^{m}$ in 2005 and $\sim 0.05^{m}$ in the early 1980s. As a second permanent light curve property we found a secondary minimum or step-like feature at phases $0.65-0.7$ which seems to be always present. When the hump amplitude is low, this feature is more pronounced, looking like an eclipselike feature corresponding to the total minimum in the whole orbital light curve. On the other hand, for a large amplitude hump $\left(>0.2^{m}\right)$ the step-like feature seems to be less pronounced.

\subsection{Search for superhumps: a new detection in 2007}

In RR Pic, the superhump phenomenon is an additional periodic modulation in the light curve with smaller amplitude than that of the orbital hump. Therefore, a search for superhumps is only possible in the residual data after subtraction of the dominant frequency. Any additional frequency found close to the orbital one could be potentially associated with a $P_{\mathrm{sh}}$.

To ensure that the residual light curves of all campaigns were not contaminated by the presence of harmonics and aliases of the orbital period, we extracted up to the sixth orbital harmonics from 
the data. In this manner, we analyse all campaigns. In Fig. 2 we show the Fourier spectra of the residuals light curves for selected campaigns which covered at least two orbital cycles. To check the possible presence of any periodic signal associated directly to the sampling, we created an artificial sample containing the same time values of each set data versus a constant magnitude value. Periodograms of those artificial data represent the individual spectral windows, which are shown as inserts in Fig. 2, centred on the orbital frequency and scaled to the residual amplitudes for each campaign. The noise spectrum was calculated as the average of the remainder amplitudes in certain frequency steps of a size defined by the box size parameter (see [7] for details) in the range from 0 to $30 \mathrm{c} / \mathrm{d}$. A signal near the orbital period that exceeds the $3 \sigma$ noise level, which corresponds to the dashed line in Fig. 2, is considered for further superhump analysis. Only in the 2005 data ([19], hereafter campaign 2005-2) and the 2007 CBA campaign we found signals according to these criteria, which are highlighted with an arrow in Fig. 2.

Although the 2005 CBA observations (campaign 2005-1) were performed only about one month before the 2005-2 campaign, we did not find any significant signal close to the 2005-2 signal $\left(f_{s h}=6.33 \pm 0.02 \mathrm{c} / \mathrm{d} ; P_{s h}=3.79 \pm 0.01 \mathrm{~h}\right.$, where we have used the actual frequency resolution of this campaign as error in frequency, i.e., $1 / \Delta t$ ). In order to check whether this null detection can be attributed to the time resolution and the coverage of the data instead of the real absence of the superhump, we folded those with ephemeris corresponding to the first minimum observed in 2005-2.

$$
H J D(\min )=2453409.54719+0^{d} .1577(4) E
$$

Comparing both averaged phased light curves, the superhump found in 2005-2 (February-April; Fig. 3 b) is clearly absent in the 2005-1 data (January; Fig. 3 a). Instead, we do find a clear detection of the superhump in the 2007 data. The residual spectrum showed several peaks close to the orbital signal. The highest peak found at $f_{s h}=6.37 \pm 0.04 \mathrm{c} / \mathrm{d}$ is equivalent to $P_{\mathrm{sh}}=3.77 \pm 0.02 \mathrm{~h}$. The other neighbouring peaks refer to one-cycle per day aliases, as is evident from the spectral window (Fig. 2). Choosing the minimum as zero point for the superhump phase yields the ephemeris

$$
H J D(\min )=2454114.07167+0^{d} .1569(6) E
$$

The corresponding phase-folded light curve is shown in Fig. 3 (c). Within the errors, this new superhump period is identical to that found by [19] in 2005, with a period excess $\varepsilon \approx 8.6$ per cent longer than the orbital period $\left(\varepsilon=\left(P_{\mathrm{sh}}-\mathrm{P}_{\text {orb }}\right) / \mathrm{P}_{\text {orb }},[13]\right)$. The superhump light curves shapes in both sets are rather similar, being slightly asymmetric with maxima around superhump phases 0.6-0.7 (Fig. 3 b and c).

\section{Discussion}

\subsection{RR Pic in context with other superhumpers}

Among all CVs which are neither dwarf novae nor AM CVn stars, positive superhumps (with $\varepsilon>0$ ) have been reported for seven classical post-novae and 17 nova-like variables. Properties for classical novae are shown in Table 1.

In this context, we investigated the role of RR Pic into the well-known close relation between $P_{\text {orb }}$ and $P_{\text {sh }}$ found first by [23]. [2] gave a new version of this relation, in the form 
Table 1: Properties of post-novae with confirmed positive superhumps.

\begin{tabular}{lrrrrc}
\hline & Name & Type & $\begin{array}{r}P_{\text {orb }} \\
(\mathrm{d})\end{array}$ & $\begin{array}{r}P_{\text {sh }} \\
(\mathrm{d})\end{array}$ & Ref. \\
\hline \hline Classical & CP Pup (1942) & $\mathrm{Na}$ & 0.06126 & 0.0625 & {$[14],[9]$} \\
Novae & V1974 Cyg & $\mathrm{Na}$ & 0.08126 & 0.08522 & {$[12],[22]$,} \\
& V630 Sgr & $\mathrm{Na}$ & 0.11793 & 0.1242 & {$[30],[10]$} \\
& V4633 Sgr & $\mathrm{Na}$ & 0.12557 & 0.1277 & {$[10],[8]$} \\
& V603 Aql (1918) & $\mathrm{Na}$ & 0.13820 & 0.14646 & {$[15],[17]$} \\
& RR Cha & $\mathrm{Na}$ & 0.14010 & 0.1444 & {$[31]$} \\
& RR Pic (1925) & $\mathrm{Nb}$ & 0.14502 & 0.1577 & {$[19]$, This work, [1] } \\
\hline
\end{tabular}

$$
P_{o r b}=a+b \cdot P_{s h}
$$

for the period range $P_{\mathrm{sh}}<120$ minutes, i.e. below the period gap between 2 and 3 hours. Most SU UMa stars are located below this gap, and the Stolz-Schoembs relation is now a popular tool for period determination because it is normally much easier to observe superhumps than any orbital variation, at least for non-eclipsing cases. [2] derived a standard deviation of only 0.53 minutes for this method to get $P_{\text {orb }}$ from equation (4.1). We have repeated the calculation of these authors including new data, and extended it for cases with $P_{\mathrm{sh}}>2 \mathrm{~h}$. The results are listed in Table 2. For the dwarf novae below the period gap, slope $a$ and standard deviation ( $\sigma=0.89$ minutes) are larger in the actually available sample of 182 cases, compared to [2]. If we consider all dwarf novae, we even get $\sigma=1.32$ minutes. Nova-like stars seem to have smaller slopes than the remaining groups while the post-novae behave similar to the dwarf novae, however suffering from the small number of only seven post-novae. All groups display larger standard deviations above the period gap, compared to those of $P_{\text {orb }}<2 \mathrm{~h}$. Generally, the differences between the groups considered here are marginal, confirming that the Stolz-Schoembs relation seems to be valid for most superhumpers of any sub-type of CVs, as shown in Fig. 4, where all novae and nova-like stars are individualised, as well as the SU UMa type dwarf novae above the period gap $\left(P_{\text {orb }}>3 \mathrm{~h}\right)$.

\section{Conclusions}

We present the first systematic analysis of collected optical light curves of the classical nova RR Pic spanning 42 years, from 1972 to 2014. We found that the light curve shape of the orbital hump and its amplitude varies significantly over the different observing epochs, suggesting that the accretion disc is undergoing long-term changes. Furthermore, we corroborate the superhump detection made by [19] and additionally we find evidence for the presence of most likely the same positive superhump in $2007 \mathrm{CBA}$ data, a period $\sim 9 \%$ longer than the orbital period. Equally important was the fact that CBA data have been obtained in 2005 only one month before those by [19], but did not reveal any evidence for superhumps, implying the superhump is a repetitive, but sporadic event, arising quickly. 
Table 2: $P_{\mathrm{sh}}-P_{\mathrm{orb}}$ linear fit considering different sub-samples of CVs. $a$ and $b$ correspond to the parameters of the linear fit, $\sigma_{a}$ and $\sigma_{b}$ to the mean errors, $\sigma$ refers to the standard deviation. $N$ represents the number of stars in each sub-sample. The first row shows the fit found by [2].

\begin{tabular}{llllllr}
\hline \hline \multicolumn{7}{c}{$P_{\text {orb }}(\min )=a+b \cdot P_{\text {sh }}(\min )$} \\
& $a$ & $\sigma_{a}$ & $b$ & $\sigma_{b}$ & $\sigma$ & $N$ \\
\hline \hline Derived by [2] & & & & & & \\
$\mathrm{DN} \mathrm{P} \leq 120 \mathrm{~min}$ & 5.39 & 0.52 & 0.9162 & 0.0052 & 0.53 & 68 \\
\hline $\mathrm{DN} \mathrm{P} \leq 120 \mathrm{~min}$ & 3.66 & 0.49 & 0.9329 & 0.0053 & 0.89 & 182 \\
\hline $\mathrm{DN} \mathrm{P}>120 \mathrm{~min}$ & 14.09 & 1.62 & 0.8474 & 0.0114 & 1.90 & 35 \\
\hline $\mathrm{All} \mathrm{DN}$ & 7.09 & 0.40 & 0.8964 & 0.0038 & 1.32 & 217 \\
\hline $\mathrm{NL}$ & 17.77 & 6.14 & 0.8418 & 0.0280 & 6.26 & 17 \\
\hline $\mathrm{Na}, \mathrm{Nb}$ & 6.11 & 6.36 & 0.9193 & 0.0352 & 4.33 & 7 \\
\hline $\mathrm{NL}+(\mathrm{Na}, \mathrm{Nb})$ & 16.04 & 4.62 & 0.8531 & 0.0221 & 5.96 & 24 \\
\hline $\mathrm{All} \mathrm{CVs}$ & 7.83 & 0.42 & 0.8899 & 0.0036 & 2.32 & 241 \\
\hline \hline
\end{tabular}

We give a list of seven post-novae and 17 nova-like stars with positive superhumps recorded, and conclude that in order to determine whether the superhump phenomenon is an unusual event or common in classical novae, it is imperative to monitor post-novae systematically for long time intervals. In addition, we also determine a revised version of the Stolz-Schoembs relation between $P_{\mathrm{sh}}$ and $P_{\mathrm{orb}}$ for dwarf novae, classical novae and nova-like stars, and conclude that this relation is of general validity for all superhumpers among CVs.

This apparently also includes post-novae, with RR Pic being just one more case in a still small sub-sample. This should not come as a surprise, since it has long been suspected that the nova eruption represents a recurrent event in a CV long-term cycle [25], [20], with more recent observational evidence corroborating this hypothesis (e.g. [16], [11], [21]). The fact that postnovae present the same phenomenon as other $\mathrm{CVs}$, further strengthens our understanding of the long-term evolution of CVs.

\section{References}

[1] Fuentes-Morales I., et al., 2018, MNRAS, 474, 2493

[2] Gänsicke B. T., et al., 2009, MNRAS, 397, 2170

[3] Haefner R., Metz K., 1982, A\&A, 109, 171

[4] Hambsch F.-J., 2012, Journal of the American Association of Variable Star Observers (JAAVSO), 40, 1003

[5] Jones H. S., 1928, Nature, 121, 862

[6] Kubiak M., 1984, Acta Astron., 34, 331

[7] Lenz P., Breger M., 2005, Communications in Asteroseismology, 146, 53

[8] Lipkin Y. M., Leibowitz E. M., 2008, MNRAS, 387, 289 
[9] Mason E., et al., 2013, MNRAS, 436, 212

[10] Mróz P., et al., 2015, ApJS, 219, 26

[11] Mróz P., et al., 2016, Nature, 537, 649

[12] Olech A., 2002, Acta Astron., 52, 273

[13] Patterson J., 1998, PASP, 110, 1132

[14] Patterson J., Warner B., 1998, PASP, 110, 1026

[15] Patterson J., Kemp J., Saad J., Skillman D. R., Harvey D., Fried R., Thorstensen J. R., Ashley R., 1997, PASP, 109, 468

[16] Patterson J., et al., 2013, MNRAS, 434, 1902

[17] Peters C. S., Thorstensen J. R., 2006, PASP, 118, 687

[18] Ramsay G., Schreiber M. R., Gänsicke B. T., Wheatley P. J., 2017, A\&A, 604, A107

[19] Schmidtobreick L., Papadaki C., Tappert C., Ederoclite A., 2008, MNRAS, 389, 1345

[20] Shara M. M., Livio M., Moffat A. F. J., Orio M., 1986, ApJ, 311, 163

[21] Shara M. M., Drissen L., Martin T., Alarie A., Stephenson F. R., 2017, MNRAS, 465, 739

[22] Shugarov S. Y., Goranskij V. P., Pavlenko E. P., 2002, in Hernanz M., José J., eds, American Institute of Physics Conference Series Vol. 637, Classical Nova Explosions. pp 323-327

[23] Stolz B., Schoembs R., 1984, A\&A, 132, 187

[24] Vogt N., 1975, A\&A, 41, 15

[25] Vogt N., 1982b, ApJ, 252, 653

[26] Vogt N., Schreiber M. R., Hambsch F.-J., Retamales G., Tappert C., Schmidtobreick L., Fuentes-Morales I., 2017, PASP, 129, 014201

[27] Warner B., 1986, MNRAS, 219, 751

[28] Warner B., 1995, Cataclysmic variable stars. Cambridge Astrophysics Series, Vol. 28

[29] Whitehurst R., 1988, MNRAS, 232, 35

[30] Woudt P. A., Warner B., 2001, MNRAS, 328, 159

[31] Woudt P. A., Warner B., 2002, MNRAS, 335, 44

\section{DISCUSSION}

KENJI TANABE's Comment: 25 years ago, when I stayed at Gosha Observatory, I intended to perform the same observations of RR Pic, but I failed because of bad weather. I feel happy to know that you had success in the same observations.

IRMA FUENTES: Thanks for your comment. 


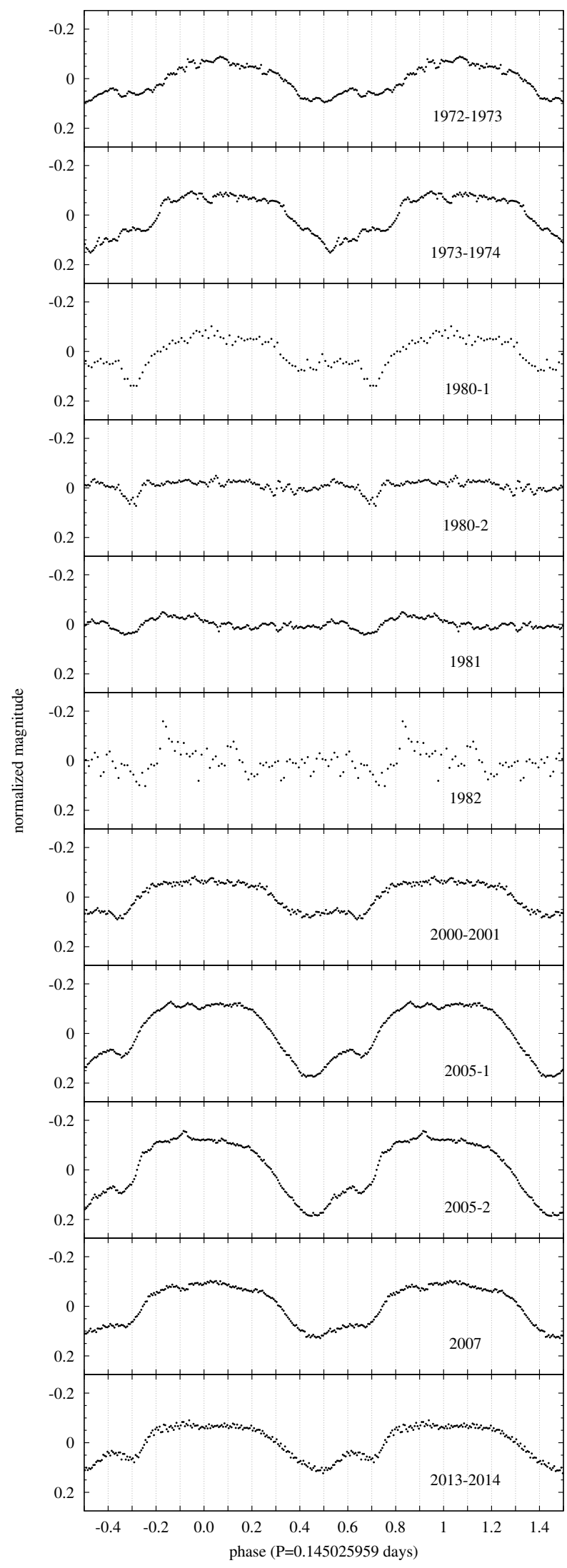

Figure 1: Orbital phase of the old nova RR Pic over the last 42 years, folded with the ephemeris given in Eq. (3.1). 

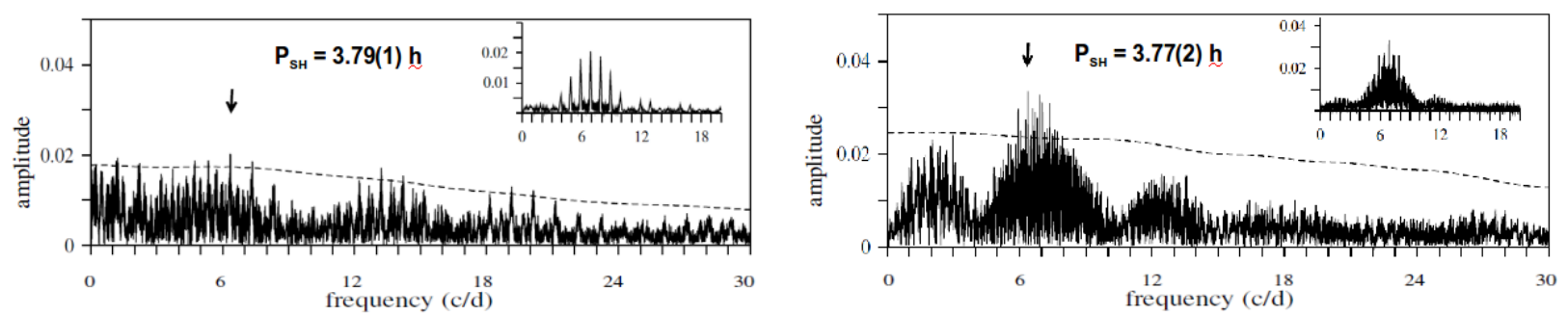

Figure 2: Periodigrams for the residual data after subtracting the orbital frequency and its harmonics for 2005 and 2007 CBA campaigns. The dashed line corresponds to the noise level of $3 \sigma$, the arrows point to suspected superhump frequencies and the inset plot shows the spectral window centred at the dominant orbital frequency.
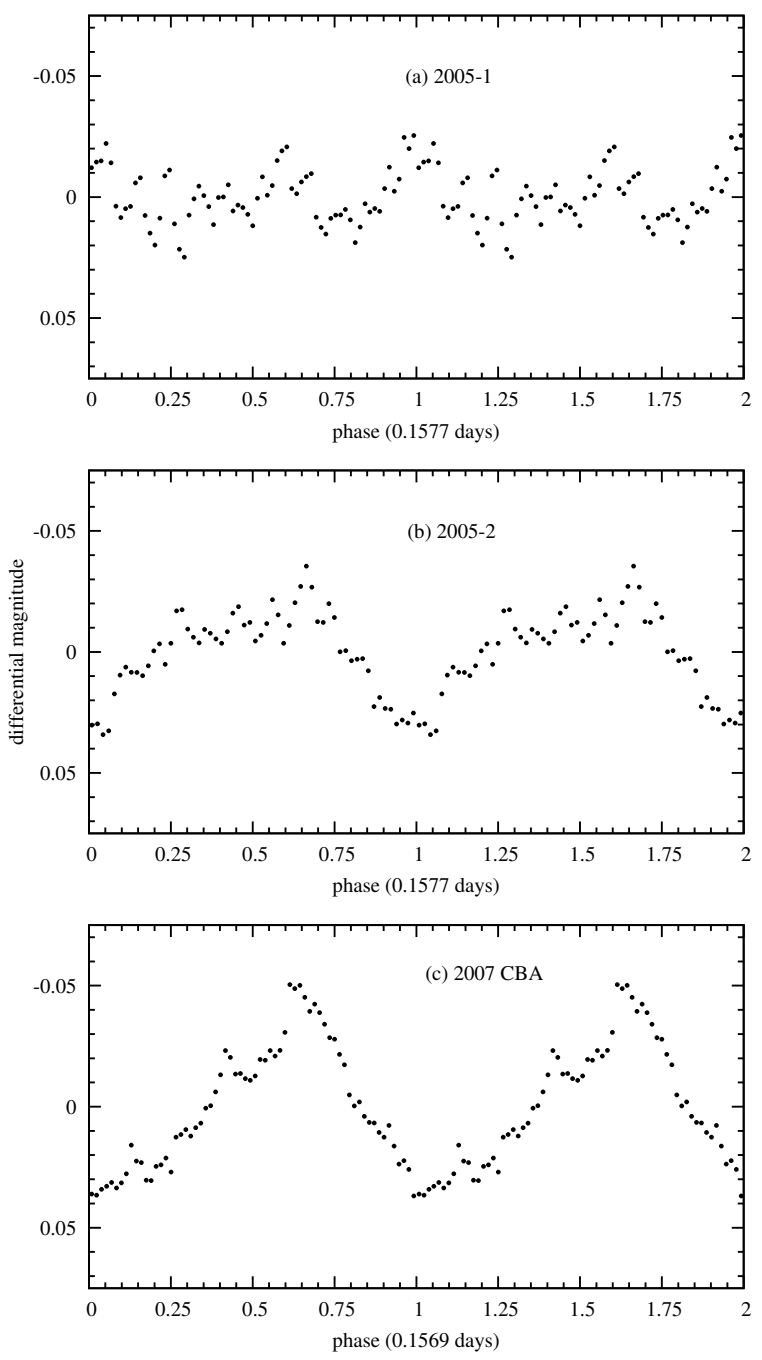

Figure 3: Superhump phase averaged into 0.02 phase bins for normalised differential magnitude light curves after subtracting the orbital frequency. (a) residuals of the 2005-1 data folded with superhump ephemeris (3.2) (b) the same but for the 2005-2 data (c) 2007 data folded with superhump ephemeris (3.3). 


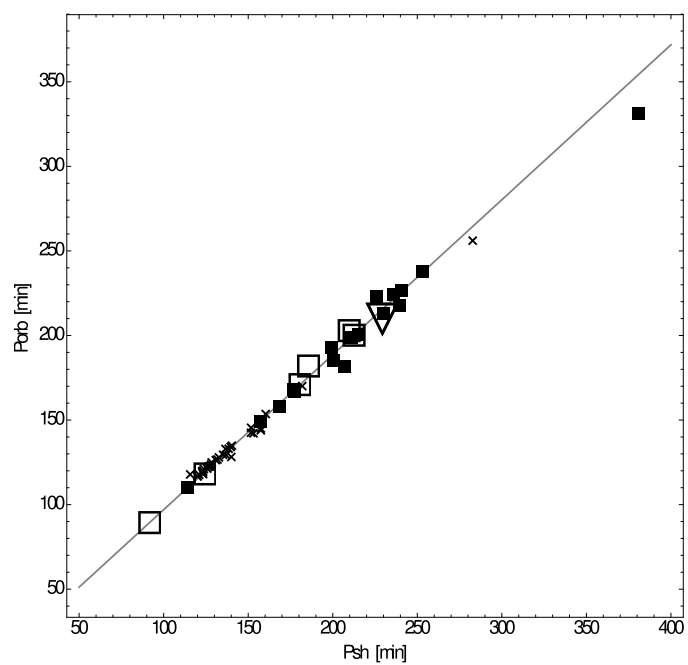

Figure 4: The Stolz-Schoembs relation $\left(P_{\mathrm{sh}}-P_{\mathrm{orb}}\right)$ for all sub-types of CVs in the entire range of $P_{\mathrm{sh}}$. Dwarf novae with $P_{\mathrm{sh}}>120 \mathrm{~min}$ are represented as crosses, all nova-like stars as filled squares, all classical novae as empty squares and the classical nova RR Pic as an inverted empty triangle. The grey line corresponds to the linear fit found by [2] for SU UMa type dwarf novae with $P_{\mathrm{sh}}<120 \mathrm{~min}$. 